\title{
The influence of a mistuned blade's staggle angle on the vibration and stability of a shaft-disk-blade assembly
}

\author{
Yi-Jui Chiu and Shyh-Chin Huang* \\ Department of Mechanical Engineering, National Taiwan University of Science and Technology, 43 Kee-Lung Rd, \\ Sec. 4, Taipei, Taiwan
}

Received 13 March 2006

Revised 2 October 2006

\begin{abstract}
The influence on coupling vibrations and stability among shaft-torsion, disk-transverse and blade-bending of a rotor system with a mistuned blade's staggle angle was investigated analytically. A shaft-disk-blade system has been found existing two types of coupling vibrations, disk-blade (DB), and blade-blade (BB) modes when the shaft was assumed rigid. If the shaft's torsional flexibility was taken into account, an additional type of coupling modes, shaft-disk-blade (SDB), appeared. When an angle-mistuned blade existed, the blades periodicity was destroyed and it was found to change not only the natural frequencies but also the types of modes. Due to blade's mistune, the shaft torsion had to participate to balance such that DB modes vanished and replaced by SDB modes. A mistuned staggle angle was numerically found to alter the natural frequencies in an almost linear trend. At last, the rotational effects were found to merge frequency loci and eventually reached an instability point. Very interestingly, a mistuned blade diminished the possible instability caused by blade-dominating modes, which existed in a perfect and periodic rotor. In words, the rotor might benefit from a mistuned blade from the stability viewpoint. The shaft-dominating mode, yet, was unaffected by the mistune and retained a possible instability.
\end{abstract}

Keywords: Coupled vibration, mistuned blade, staggle angle

\section{Introduction}

Rotor systems composed of shaft, disk and blades have been extensively applied industry. The increasing demand for higher operational speeds requires more precise tune than it used to be. Rotor dynamics has been explored over a century. Most of studies were confined to the analyses of individual components, such as disks [1-3] and blades [46]. Compound systems, such as shaft-disks [7-9] and disk-blades [10-12] were relatively limited. Recently, the demand for high speed has evoked the attention to the investigations of coupling modes among shaft, disk and blades. The coupling effects of shaft and disk were investigated, utilizing the finite element method [13,14], the transfer matrix method [15] and the assumed modes method [16]. Other studies [17-19] have addressed the effects of coupling between disk and blades. Those studies used the Rayleigh-Ritz method and the finite element method to explore the influence of the disk flexibility on the vibration characteristics and found that the disk-blades coupling frequencies approached the blade's frequencies as disk's number of nodal diameters increased. Chun and Lee [20] used assumed mode method to analyze the effect of disk flexibility on the vibrational modes of a flexible disk-blade system, and obtained more precise results, as compared to finite element method. Few studies have addressed the dynamic characteristics of a shaft-disk-blade assembly.

*Corresponding author. Tel.: +8862 27376443; Fax: +886 2 27376460; E-mail: schuang@ @ail.ntust.edu.tw. 


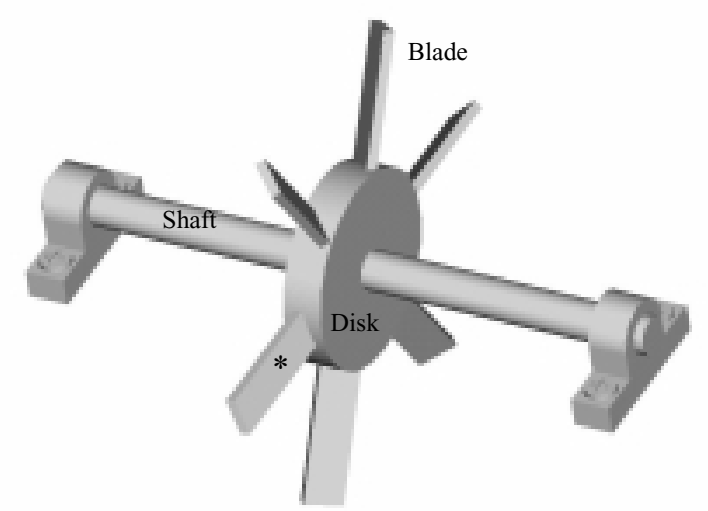

(a)

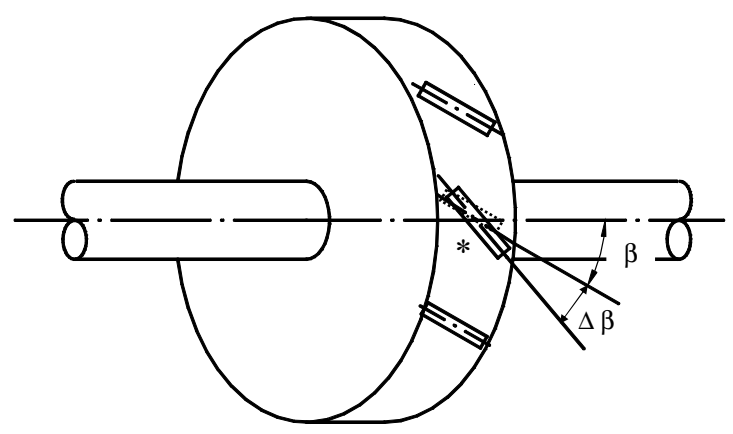

(b)

Fig. 1. (a) A shaft-disk-blade rotor with a mistuned (asterisked) blade, and (b) the mistuned angle.

Huang and Ho [21] utilized the concept of structure synthesis for a shaft-disk-blade unit. In their paper, the system was divided into two subsystems, the shaft-disk and the blades. The disk was assumed rigid and withstood the motion transmission between shaft and blades. The results showed that, there existed not only shaft-blade coupled modes but also inter-blades coupling modes. Lately, Yang and Huang [22] included disk flexibility into such a shaft-disk-blades unit and studied its free vibration and classified four types of coupling modes, shaft-disk-blade (SDB), disk-blade (DB), blade-blade (BB), and shaft-blade (SB).

The present paper extends the work of [22] but focuses on the influence of blade's mistuned angle on vibration characteristics. As well known, a perfect disk-blade assembly is symmetric and of the characteristics of a periodical structure. Any disorder or mistune, however, will destroy this periodicity. The present paper addresses the mistuned blade's staggle angle effects upon coupling vibrations among shaft, disk and blades. The instability caused by rotation and the mistuned blade is addressed as well. The mistuned error set in this paper might be exaggerated contrast to today's precision technology. Nevertheless, the present studies are to provide qualitative and quantitative overview of a periodic structure with mistune, such as rotors.

\section{Theoretical analysis}

The shaft-disk-blade assembly is shown in Fig. 1. Assume one of the blades' stagger angle (asterisked) deviates from the others, the cyclic periodicity of blades is hence destroyed. The subsequential effects such a mistuned blade are explored in this paper. The resulting from rotor investigated consists of a flexible disk, a torsional shaft and multi flexible blades fixed onto the outer edge of disk with a stagger angle $\beta$. The rotor can be decomposed into three 


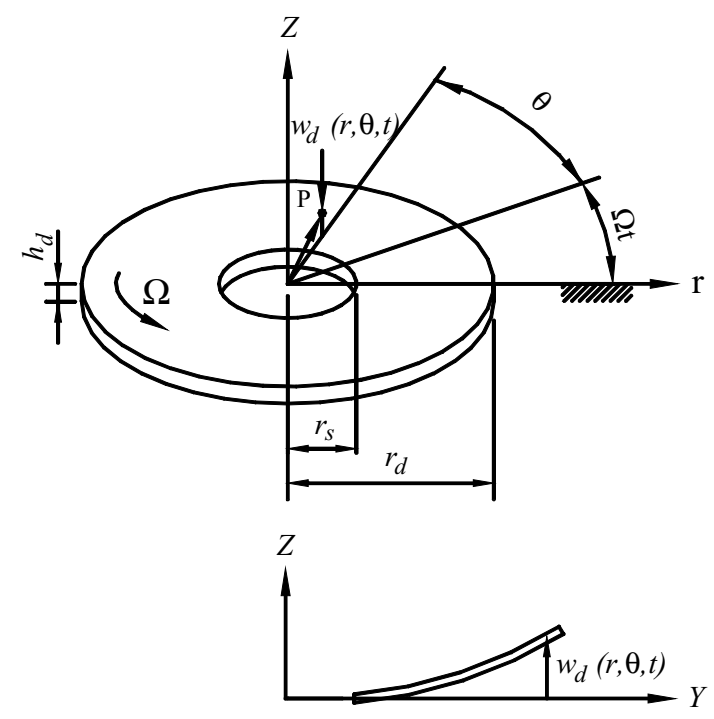

Fig. 2. The geometry and coordinates of the rotating disk.

sub-systems, torsional shaft, transverse disk and bending blades. The energies associated with components are here derived and the assumed mode method follows to discretize the equations of motion.

In the following, the blade of mistuned stagger angle is asterisked to separate from the others. It is denoted as

$$
\beta^{*}=\beta+\Delta \beta=\beta(1+\varepsilon)
$$

where $\varepsilon$ is an indication of mistuned error, positive or negative.

The torsional energies associated with the shaft-disk subsystem are:

$$
\begin{aligned}
& T_{s}=\frac{1}{2} \int_{0}^{L s} I_{s}(\dot{\phi}+\Omega)^{2} d Z+\left.\frac{I_{d}}{2}(\dot{\phi}+\Omega)^{2}\right|_{Z=Z_{d}} \\
& U_{s}=\frac{1}{2} \int_{0}^{L s} G_{s} J_{s}\left(\frac{\partial \phi}{\partial Z}\right)^{2} d Z
\end{aligned}
$$

where $\phi(Z, t)$ is the torsional displacement with respect to a constantly rotating $(\Omega)$ frame; $L_{s}, I_{s}$, and $G_{s} J_{s}$ denote the shaft's length, polar rotatary inertia, and torsional rigidity, respectively. $I_{d}$ is the disk's polar rotary inertia. The upper "dot" denotes with time derivatives. Subscripts $s$ and $d$ are designated for shaft and disk, respectively.

Figure 2 shows a disk, clamped inside and free outside, rotating at a constant speed $\Omega$. In this figure, $r_{s}$ and $r_{d}$ denote disk's inner and outer radii respectively, $h_{d}$ is disk thickness.

The energies associated with the disk in transverse vibration are

$$
\begin{aligned}
T_{d}= & \frac{\rho_{d} h_{d}}{2} \int_{r_{s}}^{r_{d}} \int_{0}^{2 \pi}\left(\dot{w}_{d}+\Omega \frac{\partial w_{d}}{\partial \theta}\right)^{2} r d \theta d r \\
U_{d}= & \frac{D}{2} \int_{r_{s}}^{r_{d}} \int_{0}^{2 \pi}\left\{\left(\nabla^{2} w_{d}\right)^{2}-(1-\nu) \frac{\partial^{2} w_{d}}{\partial r^{2}}\left[\left(\frac{1}{r} \frac{\partial w_{d}}{\partial r}+\frac{1}{r^{2}} \frac{\partial^{2} w_{d}}{\partial \theta}\right)-\left(\frac{1}{r} \frac{\partial^{2} w_{d}}{\partial r \partial \theta}-\frac{1}{r^{2}} \frac{\partial w_{d}}{\partial \theta}\right)^{2}\right]\right\} r d \theta d r \\
& +\frac{h_{d}}{2} \int_{r_{s}}^{r_{d}} \int_{0}^{2 \pi}\left[\sigma_{r}\left(\frac{\partial w_{d}}{\partial r}\right)^{2}+\sigma_{\theta}\left(\frac{1}{r} \frac{\partial w_{d}}{\partial \theta}\right)^{2}\right] r d \theta d r
\end{aligned}
$$

where $w_{d}$ is the disk's transverse displacement; $\nabla^{2}$ is the Laplacian; $D$ is the bending rigidity; $\sigma_{r}$ and $\sigma_{\theta}$ are the initial stresses in the radial and circumferential directions due to $\Omega$. These terms are of the following forms: 

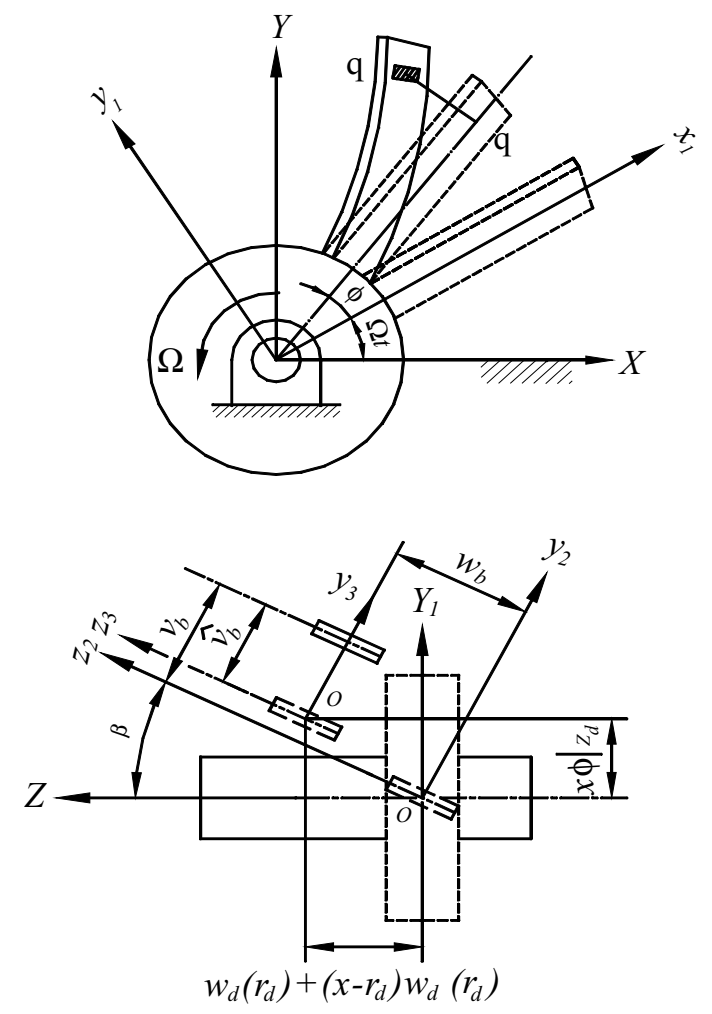

Fig. 3. Coordinate sets and deformation of a blade.

$$
\begin{aligned}
\nabla^{2} & =\frac{\partial^{2}}{\partial r^{2}}+\frac{\partial}{r \partial r}+\frac{\partial^{2}}{r^{2} \partial \theta^{2}} \\
D & =\frac{E_{d} h_{d}^{3}}{12\left(1-\nu^{2}\right)} \\
\sigma_{r} & =\frac{3+\nu}{8} \rho_{d} \Omega^{2}\left(r_{d}^{2}-r^{2}\right)+\frac{(1-\nu) \rho_{d} \Omega^{2} r_{s}^{2}\left[(3+\nu) r_{d}^{2}-(1+\nu) r_{s}^{2}\right]}{8\left[(1+\nu) r_{d}^{2}+(1-\nu) r_{s}^{2}\right]}\left(\frac{r_{d}^{2}}{r^{2}}-1\right) \\
\sigma_{\theta} & =\frac{\Omega^{2}}{8}\left[(3+\nu) r_{d}^{2}-(1+3 \nu) r^{2}\right]-\frac{(1-\nu) \rho_{d} \Omega^{2} r_{s}^{2}\left[(3+\nu) r_{d}^{2}-(1+\nu) r_{s}^{2}\right]}{8\left[(1+\nu) r_{d}^{2}+(1-\nu) r_{s}^{2}\right]}\left(\frac{r_{d}^{2}}{r^{2}}+1\right)
\end{aligned}
$$

Figure 3 shows a typical rotating blade cantilevered onto a flexible disk with a stagger angle $(\beta)$. The $(X, Y, Z)$ coordinate system is the inertia frame; $\left(x_{1}, y_{1}, z_{1}\right)$ frame rotates at a constant speed $\Omega$. $\left(x_{2}, y_{2}, z_{2}\right)$ frame turns an angle " $\beta$ " relative to $\left(x_{1}, y_{1}, z_{1}\right)$ frame, and $\left(x_{3}, y_{3}, z_{3}\right)$ frame is fixed to the blade's root.

The kinetic and strain energies associated with a blade are

$$
\begin{aligned}
T_{b}= & \frac{1}{2} \int_{r_{d}}^{r_{b}} \rho_{b} A_{b}\left\{\dot{v}_{b}^{2}+\dot{w}_{b}^{2}+\left(v_{b} \cos \beta+w_{b} \cos \beta\right)^{2} \Omega^{2}+x^{2} \Omega^{2}+2 x\left(\dot{v}_{b} \sin \beta+\dot{w}_{b} \sin \beta\right) \Omega\right\} d x \\
& +\frac{1}{2} \int_{r_{d}}^{r_{b}} I_{b}\left(\Omega \cos \beta+\frac{\partial \dot{v}_{b}}{\partial x}\right)^{2} d x \\
U_{b}= & \int_{r_{d}}^{r_{b}} \frac{E_{b} I_{A}}{2}\left(\frac{\partial^{2} v_{b}}{\partial x^{2}}\right)^{2} d x+\int_{r_{d}}^{r_{b}} \frac{1}{4} \Omega^{2} \rho_{b} A_{b}\left(r_{b}^{2}-x^{2}\right)\left[\left(\frac{\partial v_{b}}{\partial x}\right)^{2}+\left(\frac{\partial w_{b}}{\partial x}\right)^{2}\right] d x
\end{aligned}
$$


where $v_{b}$ and $w_{b}$ are the transverse displacements of the blade in $y_{2}$ and $z_{2}$ directions respectively. $I_{A}$ is the area moment of inertia about the $z_{3}$ axis, and $I_{b}$ is the polar moment of inertia. Note that $v_{b}(x, t)$ and $w_{b}(x, t)$ are contributed by the shaft's torsional displacement $\phi\left(Z_{d}, t\right)$, the disk's transverse displacement $w_{d}$, and the blade's bending displacement $\hat{v}_{b}(x, t)$, i.e.,

$$
\begin{aligned}
& v_{b}(x, t)=\hat{v}_{b}+\left.x \phi\right|_{Z_{d}} \cos \beta-\left(\left.w_{d}\right|_{r_{d}}+\left.x w_{d}^{\prime}\right|_{r_{d}}\right) \sin \beta \\
& w_{b}(x, t)=\left.x \phi\right|_{Z_{d}} \sin \beta+\left(\left.w_{d}\right|_{r_{d}}+\left.x w_{d}^{\prime}\right|_{r_{d}}\right) \cos \beta
\end{aligned}
$$

Note that the blades are assumed identical except the mistuned one and for which the $\beta$ is replaced by $\beta^{*}$. In the following, without loss of generality the mistuned blade is numbered the first blade.

Assumed mode method is adopted to discretize the continuous system, i.e.,

$$
\begin{aligned}
& \phi(Z, t)=\sum_{i=1}^{n_{s}} \Phi_{i}(Z) \eta_{i}(t)=\Phi(Z) \eta(t) \\
& w_{d}(r, \theta, t)=\sum_{i=1}^{n_{d}}\left[W_{i}^{c}(r, \theta) \zeta_{i}^{c}(t)+W_{i}^{s}(r, \theta) \zeta_{i}^{s}(t)\right]=\boldsymbol{W}(r, \theta) \zeta(t) \\
& \hat{v}_{b_{k}}(x, t)=\sum_{i=1}^{n_{b}} V_{i}(x) \xi_{i k}(t)=\boldsymbol{V}(x) \xi_{k}(t), k=1,2, \ldots, N
\end{aligned}
$$

where $\Phi_{i},\left\{W_{i}^{c}, W_{i}^{s}\right\}$ and $V_{i}$ are the mode shapes of a torsional shaft, of a transverse disk, and of a bending blade, respectively. These modes are chosen to be

$$
\begin{aligned}
& \Phi_{i}(Z)=\sin \left[\frac{(2 i-1) \pi Z}{2 L_{s}}\right] \\
& W_{i}^{c}(r, \theta)=R_{i}(r) \cos [(i-1) \theta] \\
& W_{i}^{s}(r, \theta)=R_{i}(r) \sin [(i-1) \theta]
\end{aligned}
$$

where $R_{i}(r)$ is the disk's radial function and is chosen the beam function [23].

$$
V_{i}(x)=\left(\sin \tau_{i} x-\sinh \tau_{i} x\right)+\alpha_{i}\left(\cos \tau_{i} x-\cosh \tau_{i} x\right)
$$

similar to $R_{i}(r)$, is a beam function for blade with

$$
\begin{gathered}
{\left[\cos \tau_{i}\left(r_{b}-r_{d}\right)\right]\left[\cosh \tau_{i}\left(r_{b}-r_{d}\right)\right]+1=0} \\
\alpha_{i}=\frac{-\sin \tau_{i}\left(r_{b}-r_{d}\right)-\sinh \tau_{i}\left(r_{b}-r_{d}\right)}{\cos \tau_{i}\left(r_{b}-r_{d}\right)+\cosh \tau_{i}\left(r_{b}-r_{d}\right)}
\end{gathered}
$$

$\eta_{i}, \zeta_{i}^{c}, \zeta_{i}^{s}$ and $\xi_{i k}$ are the participation factors. $n$ 's with subscripts for the corresponding subsystems, are the number of modes deemed necessary for required accuracy. In this study, $\left(n_{s}, n_{d}, n_{b}\right)=(7,9,10)$ assures the convergence to $10^{-3} \mathrm{~Hz}$.

Substitution of the above equations into the energy expressions and employing the Lagrange equations yields the equations of motion in matrix notation as

$$
[M] \ddot{\boldsymbol{q}}-\Omega[P] \dot{\boldsymbol{q}}+\left(\left[K^{e}\right]+\left[K^{i}\right]-\Omega^{2}\left[K^{\Omega}\right]\right) \boldsymbol{q}=\mathbf{0}
$$

where $-\Omega[P]$, generated from the Coriolis effect, induces the bifurcation of the natural frequencies [22]. Closer inspection of $[P]$ reveals that it is nonzero only if the disk is flexible. In words, it is the disk flexibility that causes frequency bifurcation. $\left[K^{e}\right]$, arising from the elastic deflection, dominates at low rotational speed. $\left[K^{i}\right]$ is the stiffness due to initial stress resulted form rotation. The term $-\Omega^{2}\left[K^{\Omega}\right]$ softens the rotor and becomes very significant 
at high rotational speed. It is also the major role that decides rotor's stability. The matrices $[M],[P],\left[K^{e}\right],\left[K^{i}\right]$ and $\left[K^{\Omega}\right]$ are given as:

$$
\begin{aligned}
& {[M]=\left[\begin{array}{llllll}
{\left[M_{s}\right]_{n_{s} \times n_{s}}} & {[0]_{n_{s} \times 2 n_{d}}} & {\left[M_{s b}^{*}\right]_{n_{s} \times n_{b}}} & {\left[M_{s b}\right]_{n_{s} \times n_{b}}} & \cdots & {\left[M_{s b}\right]_{n_{s} \times n_{b}}} \\
{[0]_{2 n_{d} \times n_{s}}^{T}} & {\left[M_{d}\right]_{2 n_{d} \times 2 n_{d}}\left[M_{d b}^{*}\right]_{2 n_{d} \times n_{b}}\left[M_{d b}\right]_{2 n_{d} \times n_{b}}} & \cdots & {\left[M_{d b}\right]_{2 n_{d} \times n_{b}}} \\
{\left[M_{s b}^{*}\right]_{n_{b} \times n_{s}}^{T}\left[M_{d b}^{*}\right]_{n_{b} \times n_{d}}^{T}\left[M_{b}^{*}\right]_{n_{b} \times n_{b}}} & {[0]_{n_{b} \times n_{b}}} & \cdots & {[0]_{n_{b} \times n_{b}}} \\
{\left[M_{s b}\right]_{n_{b} \times n_{s}}^{T}\left[M_{d b}\right]_{n_{b} \times n_{d}}^{T}} & {[0]_{n_{b} \times n_{b}}^{T}} & {\left[M_{b}\right]_{n_{b} \times n_{b}}} & \ddots & \vdots \\
\vdots & \vdots & \vdots & \ddots & \ddots & {[0]_{n_{b} \times n_{b}}} \\
{\left[M_{s b}\right]_{n_{b} \times n_{s}}^{T}} & {\left[M_{d b}\right]_{n_{b} \times n_{d}}^{T}} & {[0]_{n_{b} \times n_{b}}^{T}} & \ldots & {[0]_{n_{b} \times n_{b}}^{T}\left[M_{b}\right]_{n_{b} \times n_{b}}}
\end{array}\right]} \\
& {[P]=\left[\begin{array}{llllll}
{[0]_{n_{s} \times n_{s}}} & {[0]_{n_{s} \times 2 n_{d}}} & {[0]_{n_{s} \times n_{b}}} & \cdots & \cdots & {[0]_{n_{s} \times n_{b}}} \\
{[0]_{2 n_{d} \times n_{s}}\left[P_{d}\right]_{2 n_{d} \times 2 n_{d}}} & {[0]_{2 n_{d} \times n_{b}}} & \cdots & \cdots & {[0]_{2 n_{d} \times n_{b}}} \\
{[0]_{n_{b} \times n_{s}}^{T}} & {[0]_{n_{b} \times 2 n_{d}}^{T}} & {[0]_{n_{b} \times n_{b}}} & {[0]_{n_{b} \times n_{b}}} & \cdots & {[0]_{n_{b} \times n_{b}}} \\
\vdots & \vdots & {[0]_{n_{b} \times n_{b}}} & \ddots & \ddots & \vdots \\
\vdots & \vdots & \vdots & \ddots & \ddots & {[0]_{n_{b} \times n_{b}}} \\
{[0]_{n_{b} \times n_{s}}^{T}} & {[0]_{n_{b} \times 2 n_{d}}^{T}} & {[0]_{n_{b} \times n_{b}}} & \cdots & {[0]_{n_{b} \times n_{b}}[0]_{n_{b} \times n_{b}}}
\end{array}\right]} \\
& {\left[K^{e}\right]=\left[\begin{array}{llllll}
{\left[K_{s}^{e}\right]_{n_{s} \times n_{s}}} & {[0]_{n_{s} \times 2 n_{d}}} & {[0]_{n_{s} \times n_{b}}} & \ldots & \ldots & {[0]_{n_{s} \times n_{b}}} \\
[0]]_{n_{n} \times n_{s}} & {\left[K_{d}^{e}\right]_{2 n_{d} \times 2 n_{d}}[0]_{2 n_{d} \times n_{b}}} & \ldots & \ldots & {[0]_{2 n_{d} \times n_{b}}} \\
[0]]_{n_{b} \times n_{s}}^{T} & {[0]_{n_{b} \times 2 n_{d}}^{T}} & {\left[K_{b}^{e *}\right]_{n_{b} \times n_{b}}[0]_{n_{b} \times n_{b}}} & \ldots & {[0]_{n_{b} \times n_{b}}} \\
\vdots & \vdots & {[0]_{n_{b} \times n_{b}}} & {\left[K_{b}^{e}\right]_{n_{b} \times n_{b}}} & \ddots & \vdots \\
\vdots & \vdots & \vdots & \ddots & \ddots & {[0]_{n_{b} \times n_{b}}} \\
{[0]_{n_{b} \times n_{s}}^{T}} & {[0]_{n_{b} \times 2 n_{d}}^{T}} & {[0]_{n_{b} \times n_{b}}} & \ldots & {[0]_{n_{b} \times n_{b}}\left[K_{b}^{e}\right]_{n_{b} \times n_{b}}}
\end{array}\right]} \\
& {\left[K^{i}\right]=\left[\begin{array}{llllll}
{[0]_{n_{s} \times n_{s}}} & {[0]_{n_{s} \times 2 n_{d}}} & {[0]_{n_{s} \times n_{b}}} & \cdots & \cdots & {[0]_{n_{s} \times n_{b}}} \\
{[0]_{2 n_{d} \times n_{s}}^{T}} & {\left[K_{d}^{i}\right]_{2 n_{d} \times 2 n_{d}}[0]_{2 n_{d} \times n_{b}}} & \cdots & \cdots & {[0]_{2 n_{d} \times n_{b}}} \\
[0]]_{n_{b} \times n_{s}}^{T} & {[0]_{n_{b} \times 2 n_{d}}^{T}} & {[0]_{n_{b} \times n_{b}}} & {[0]_{n_{b} \times n_{b}}} & \cdots & {[0]_{n_{b} \times n_{b}}} \\
\vdots & \vdots & {[0]_{n_{b} \times n_{b}}} & \ddots & \ddots & \vdots \\
\vdots & \vdots & \vdots & \ddots & \ddots & {[0]_{n_{b} \times n_{b}}} \\
{[0]_{n_{b} \times n_{s}}^{T}} & {[0]_{n_{b} \times 2 n_{d}}^{T}} & {[0]_{n_{b} \times n_{b}}} & \cdots & {[0]_{n_{b} \times n_{b}}[0]_{n_{b} \times n_{b}}}
\end{array}\right]} \\
& {\left[K^{\Omega}\right]=\left[\begin{array}{llllll}
{\left[K_{s}^{\Omega}\right]_{n_{s} \times n_{s}}} & {[0]_{n_{s} \times 2 n_{d}}} & \left.\left[K_{s b}^{\Omega *}\right]_{n_{s} \times n_{b}}\left[K_{s b}^{\Omega}\right]\right]_{n_{s} \times n_{b}} & \ldots & {\left[K_{s b}^{\Omega}\right]_{n_{s} \times n_{b}}} \\
[0]]_{2 n_{d} \times n_{s}}^{T} & {\left[K_{d}^{\Omega}\right]_{2 n_{d} \times 2 n_{d}}[0]_{2 n_{d} \times n_{b}}} & \ldots & \ldots & [0]]_{2 n_{d} \times n_{b}} \\
{\left[K_{s b}^{\Omega *}\right]_{n_{b} \times n_{s}}^{T}[0]_{n_{b} \times 2 n_{d}}^{T}} & {\left[K_{b}^{\Omega *}\right]_{n_{b} \times n_{b}}} & {[0]_{n_{b} \times n_{b}}} & \ldots & {[0]_{n_{b} \times n_{b}}} \\
{\left[K_{s b}^{\Omega}\right]_{n_{b} \times n_{s}}^{T}} & \vdots & {[0]_{n_{b} \times n_{b}}} & {\left[K_{b}^{\Omega}\right]_{n_{b} \times n_{b}}} & \ddots & \vdots \\
\vdots & \vdots & \vdots & \ddots & \ddots & {[0]_{n_{b} \times n_{b}}} \\
{\left[K_{s b}^{\Omega}\right]_{n_{b} \times n_{s}}^{T}} & {[0]_{n_{b} \times 2 n_{d}}^{T}} & {[0]_{n_{b} \times n_{b}}} & \cdots & {[0]_{n_{b} \times n_{b}}\left[K_{b}^{\Omega}\right]_{n_{b} \times n_{b}}}
\end{array}\right]}
\end{aligned}
$$

The dimensions of the above matrices are $\left(n_{s}+2 n_{d}+N \times n_{b}\right) \times\left(n_{s}+2 n_{d}+N \times n_{b}\right)$, where are denoting the number of blades. The entries of the above matrices are given in the Appendix. If the mistune is zero, the system returns to a periodic structure and it is the base for comparison in the following studies. $\boldsymbol{q}$ is a generalized vector, i.e.,

$$
\boldsymbol{q}=\left\{\eta^{T} \backslash \zeta^{c T} \zeta^{s T} \backslash \xi_{1}^{T} \xi_{2}^{T} \cdots \xi_{N}^{T}\right\}^{T}
$$

In the usual manner for free vibration analysis, it is assumed the solution is of the form $\boldsymbol{q}=\{c\} e^{\lambda t}$, with $\{c\}$ the undetermined coefficient vector and $\lambda$ the eigenvalue. Note that $\lambda$ is pure imaginary for most of the undamped rotors, 
Table 1

Geometric and material properties of the illustrated examples

\begin{tabular}{lll}
\hline Shaft & density: $\rho_{s}$ & $7850 \mathrm{~kg} / \mathrm{m}^{3}$ \\
& shear modulus: $G_{s}$ & $75 \mathrm{Gpa}$ \\
& shaft length: $L_{s}$ & $0.6 \mathrm{~m}$ \\
& radius: $r_{s}$ & $0.04 \mathrm{~m}$ \\
Disk & density: $\rho_{d}$ & $7850 \mathrm{~kg} / \mathrm{m}^{3}$ \\
& Young's modulus: $E_{d}$ & $200 \mathrm{Gpa}$ \\
& location: $z_{d}$ & $0.3 \mathrm{~m}$ \\
& outer radius: $r_{d}$ & $0.2 \mathrm{~m}$ \\
& thickness: $h_{d}$ & $0.03 \mathrm{~m}$ \\
& Poisson's ratio: $\nu$ & 0.3 \\
Blade & density: $\rho_{b}$ & $7850 \mathrm{~kg} / \mathrm{m}^{3}$ \\
& Young's modulus: $E_{b}$ & $200 \mathrm{Gpa}$ \\
& blade outer end: $r_{b}$ & $0.4 \mathrm{~m}$ \\
& cross-section: $A_{b}$ & $1.2 \times 10^{-4} \mathrm{~m}^{2}$ \\
& area moment of inertia: $I_{A}$ & $1.92 \times 10^{-9} \mathrm{~m}^{2}$ \\
& Staggle angle: $\beta$ & $60^{\circ}$ \\
Rotational speed: $\Omega$ & $0 \sim 123000 \mathrm{rpm}$ \\
\hline
\end{tabular}

Table 2

Natural frequencies $(\mathrm{Hz})$ of shaft-disk, disk and clamped blade

\begin{tabular}{lccc}
\hline Component's n.f. & $\omega_{1}$ & $\omega_{2}$ & $\omega_{3}$ \\
\hline shaft-disk (w/o blades) & 207.418 & 2645.690 & 5267.204 \\
Disk (shaft rigid ) & 921.227 & 974.922 & 1205.039 \\
clamped blade (shaft-disk rigid) & 81.538 & 510.99 & 1430.788 \\
\hline
\end{tabular}

i.e., $\lambda=j \omega, j=\sqrt{-1}$, unless the rotor reaches threshold speed of instability, where $\lambda=a+j \omega$. Equation (23), then yields to be:

$$
\left\{\left(\left[K^{e}\right]+\left[K^{i}\right]-\Omega^{2}\left[K^{\Omega}\right]\right)-\lambda \Omega[P]+\lambda^{2}[M]\right\}\{c\}=\{0\}
$$

The characteristic equation is

$$
\left|\left(\left[K^{e}\right]+\left[K^{i}\right]-\Omega^{2}\left[K^{\Omega}\right]\right)-\lambda \Omega[P]+\lambda^{2}[M]\right|=0
$$

\section{Numerical results}

To avoid dimensional dependence, all the numerical results are normalized with respect to the cantilevered blade's first natural frequency $\left(\omega_{b 1}\right)$, i.e., $\omega^{*}=\omega / \omega_{b 1}$ and $\Omega^{*}=\Omega / \omega_{b 1}$. Table 1 lists the geometric and material properties of the illustrated examples. Note that, the length of blades are deliberately elongated in order to magnify the coupling behaviors. Table 2 gives the natural frequencies of individual components, with the other components either temporarily removed or assumed rigid. Table 2 provides a comparison basis for the effects of component flexibility on coupling vibration. These frequencies serve as validation and interpretation of the numerical results as well.

The first interest arises from wondering the effects of shaft's torsional flexibility, which was frequently ignored on discussing the coupling vibrations. Figure 4 shows the frequency shifts due to shaft's torsional flexibility for a sixand a seven-blade rotor. Note that the abscissa, not drawn in a linear scale, has three reference marks respectively at $\omega^{*}=1,2.544$ and 6.267 denoting blade's first bending, shaft's first torsion, and blade's second bending frequency (ref. Table 1). For a rotor of rigid shaft, the coupling modes (hollow symbols) could be grouped into two categories, the disk-blade coupling (DB), and blade-blade coupling (BB). Once the shaft's flexibility is included, there are three types of coupling modes, shaft-disk-blade (SDB), disk-blade (DB), and blade-blade (BB). In the six-blade case, 1a (SDB) mode is evolved from the original fouth DB mode and becomes the lowest mode due to shaft's flexibility. $1 \mathrm{~g}$ (SDB) mode, where shaft's torsion predominates, is a generated new mode. The BB modes are of repeated 


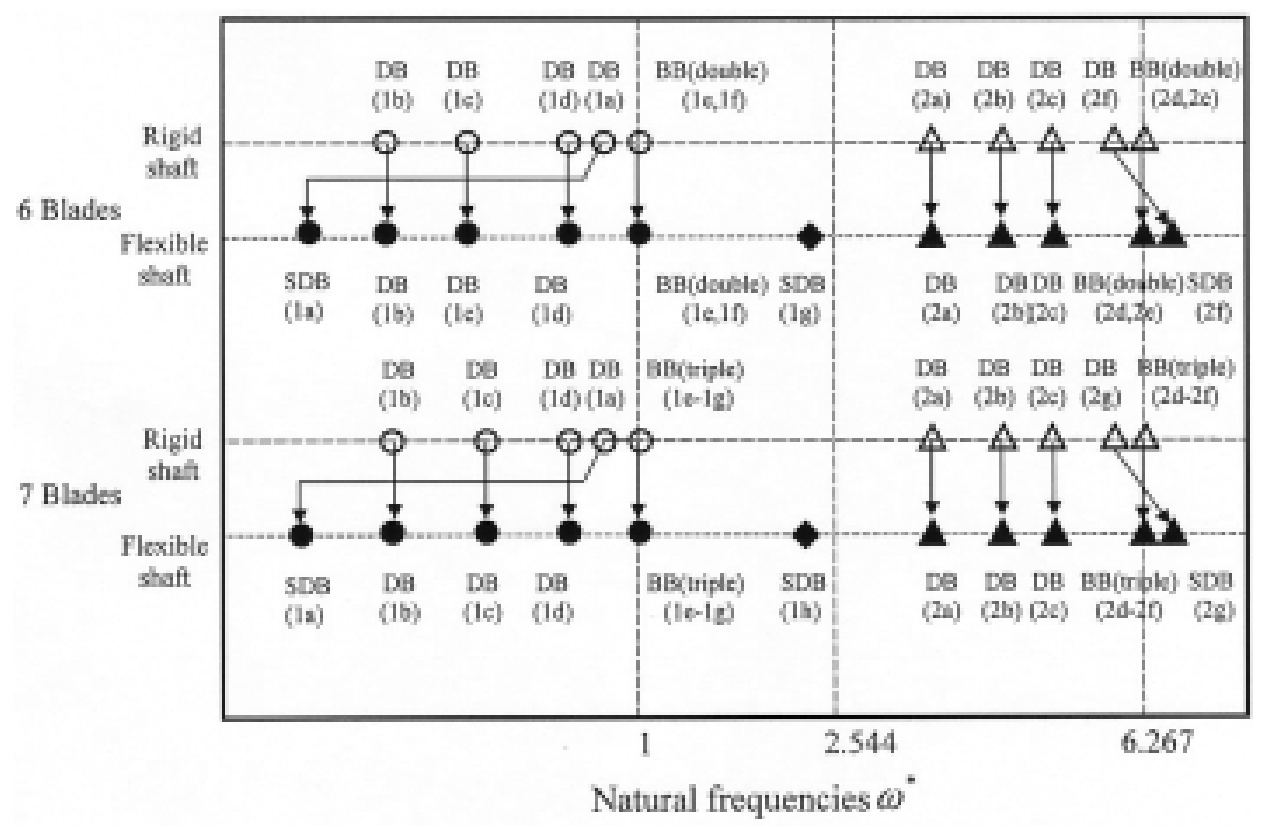

Fig. 4. Frequency changes due to shaft's flexibility for a six- and a seven-blade rotor. second mode predominates, $\$$ Shaft's first torsional mode predominates.

frequencies. $2 \mathrm{f}$ (DB) mode changes to SDB mode but its frequency is higher than BB modes, unlike 1a mode. As to the seven-blade case, very similar phenomenon appears except different multiplicity in BB modes. It has been concluded, from numerical results [22], that BB modes were of $(N-1) / 2$ and $(N-2) / 2$ multiplicity for odd and even number blades.

Figure 5 enhances the previous interpretations and illustrates the mode shapes of a six-blade system. The first x-y plot denotes the shaft's torsional displacement. The disk's and blade's deflections are illustrated in the following diagrams. Each mode's natural frequency and coupling type are indicated on each plot's upper left and right. Figure 5 demonstrates the mode shapes of a well-tuned system, which are used as references to a corresponding mistuned system. From the torsional displacement plot, one notices that only $1 \mathrm{a}$ and $1 \mathrm{~g}$ are shaft involved modes. 1b-1d modes are disk and blades (DB) related modes and 1e-1f are inter-blades (BB) modes.

Figure 6 shows the frequency changes due to a mistuned blade for a six- and a seven-blade system. The mistuned staggle angle error is set 5\%. According to Fig. 4, there are three types of coupling modes in a tuned system, said shaft-disk-blade (SDB), disk-blade (DB), and blade-blade (BB). With a mistune, there exist only two types of coupling modes, shaft-disk-blade (SDB), and blade-blade (BB). The DB modes do not appear any longer due to the mistune destroying blades' symmetry such that the shaft must participate to balance the blades forces.

Figure 7 shows the first six modes of a mistuned six-blades system. The torsional plot verifies that except the BB modes the shaft deforms with the blades. This figure reveals a very significant phenomenon, as compared to Fig. 5, i.e., the original DB modes ( $1 \mathrm{~b}, 1 \mathrm{c}, 1 \mathrm{~d}$, etc.) evolve to the SDB modes. As to the BB modes, the frequencies remain. It is due to BB modes always occurred at repeated frequencies and the mode can orient itself such that the mistuned blade experiences no deflection, as shown in Fig. 7 no deflection on the asterisked blade in 1e, 1f modes. Mode $1 \mathrm{~g}$ where torsional mode predominates, retains an SDB mode but with a slightly lower frequency. Figure 8 illustrates how the modes frequencies vary with the blade's mistuned angle. First, to both sides the DB modes switch to SDB modes as depicted previously. Second, the numerical results showed that the frequency changed almost linearly with the staggle angle error except the BB modes, which remain the same due to no deformation at mistuned blade.

At last, the system's natural frequencies varying with rotation are illustrated. Figure 9 plots the natural frequencies of a well-tuned six-blade system. From Fig. 9(a), it is seen there exist bifurcation for each mode. The forward (positive) and backward (negative) frequencies approach to each other with the increase of rotation. Eventually these two frequencies merge and become one. At the merge, it implies a possible instability. Figure 9(b) draws the real 

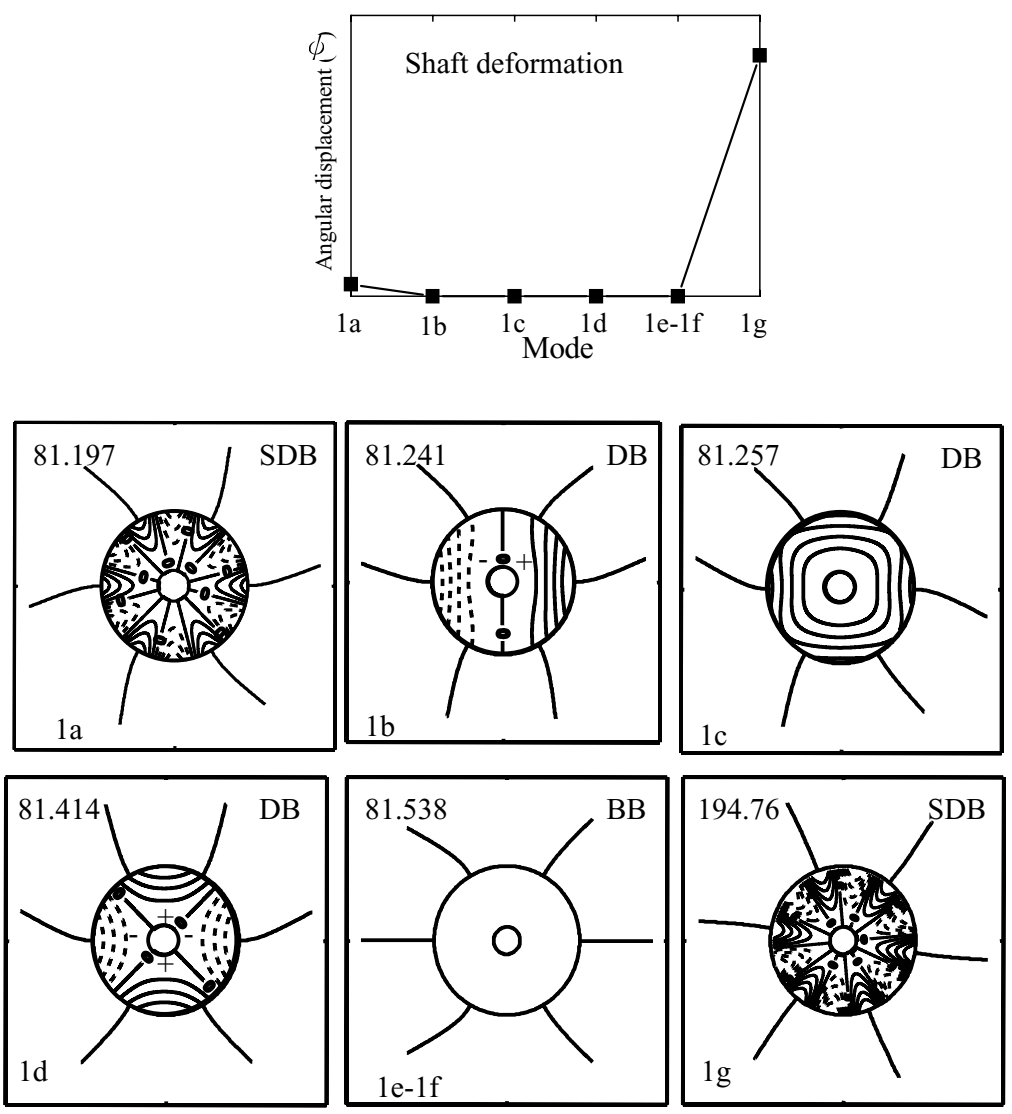

Fig. 5. The first six modes of the six-blade rotor.

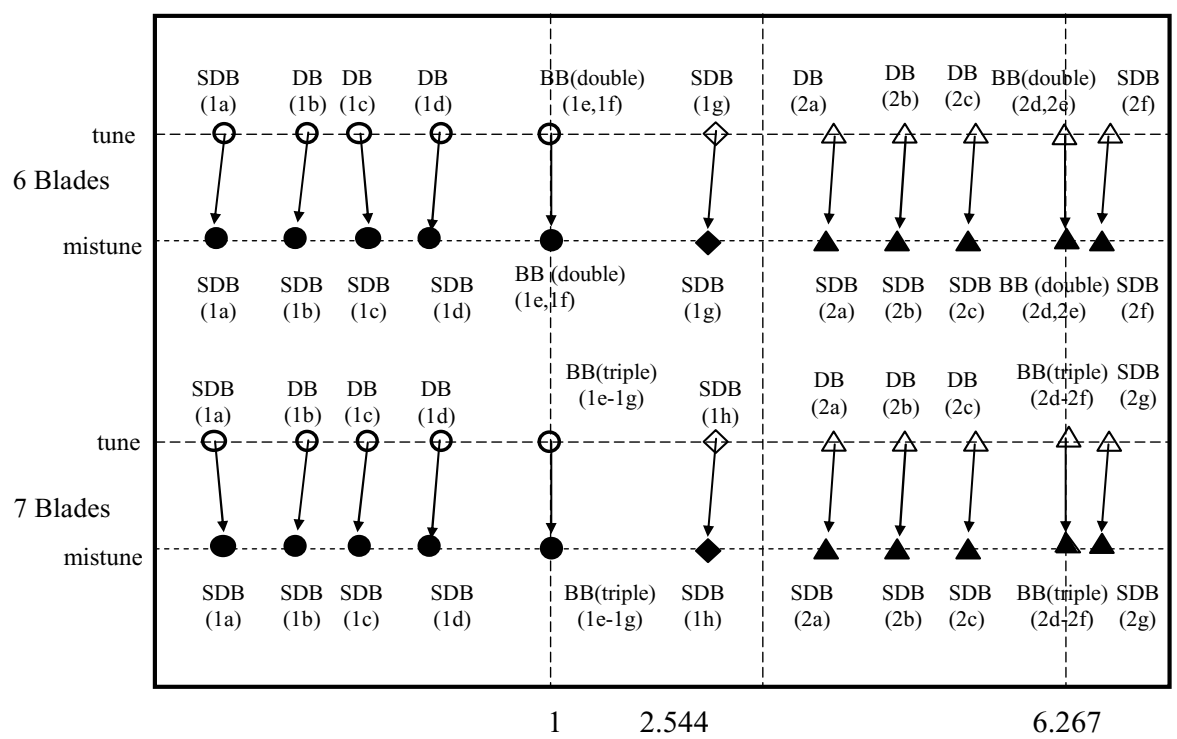

Natural frequencies $\omega^{*}$

Fig. 6. Frequency changes due to a mistuned blade in a six- and a seven-blade rotor.

Blades' first mode predominates, $\triangle \boldsymbol{\Delta}$ Blades' second mode predominates, $\diamond \diamond$ Shaft's first torsional mode predominates. 

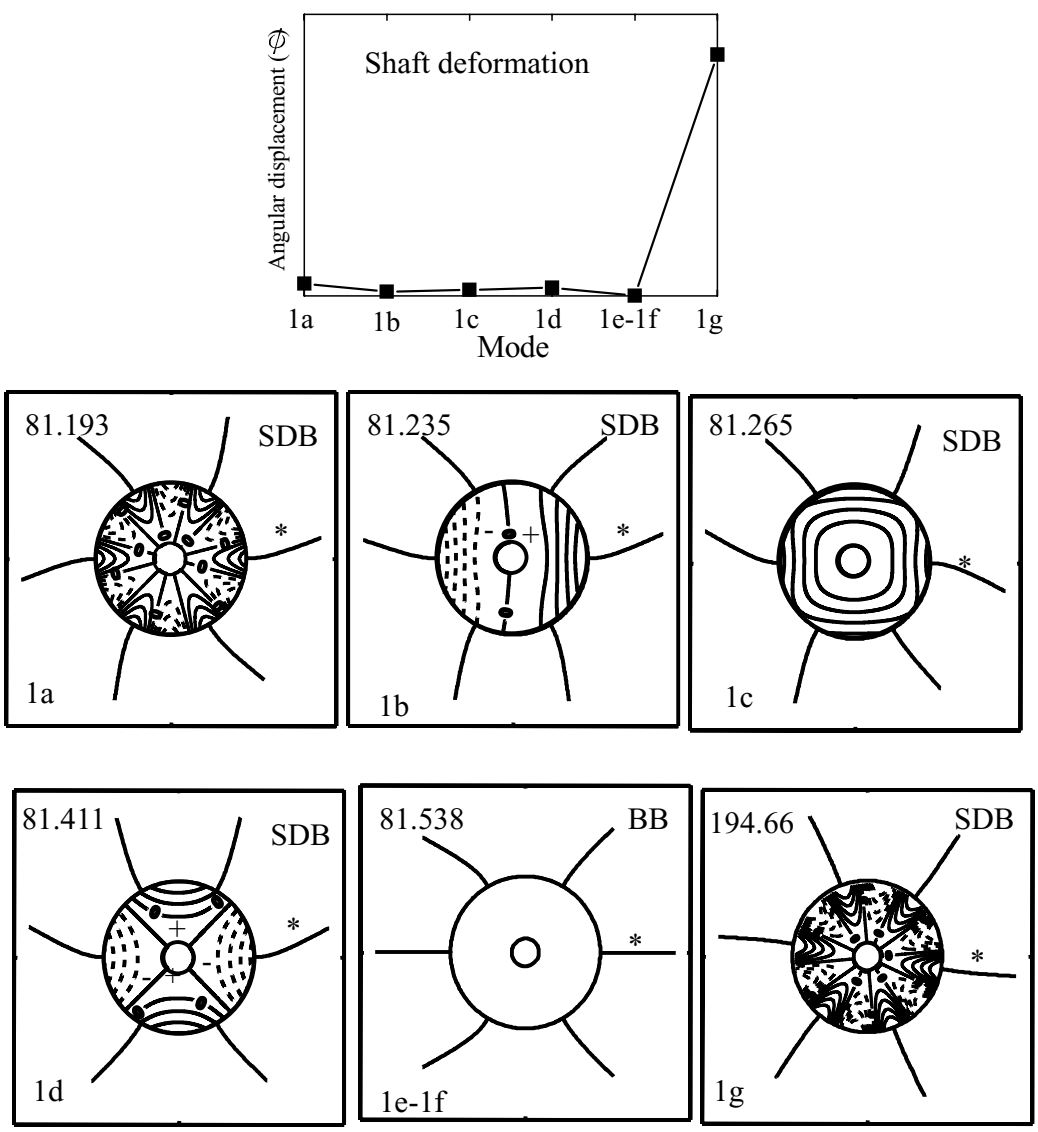

Fig. 7. The first six modes of the mistuned six-blade rotor.

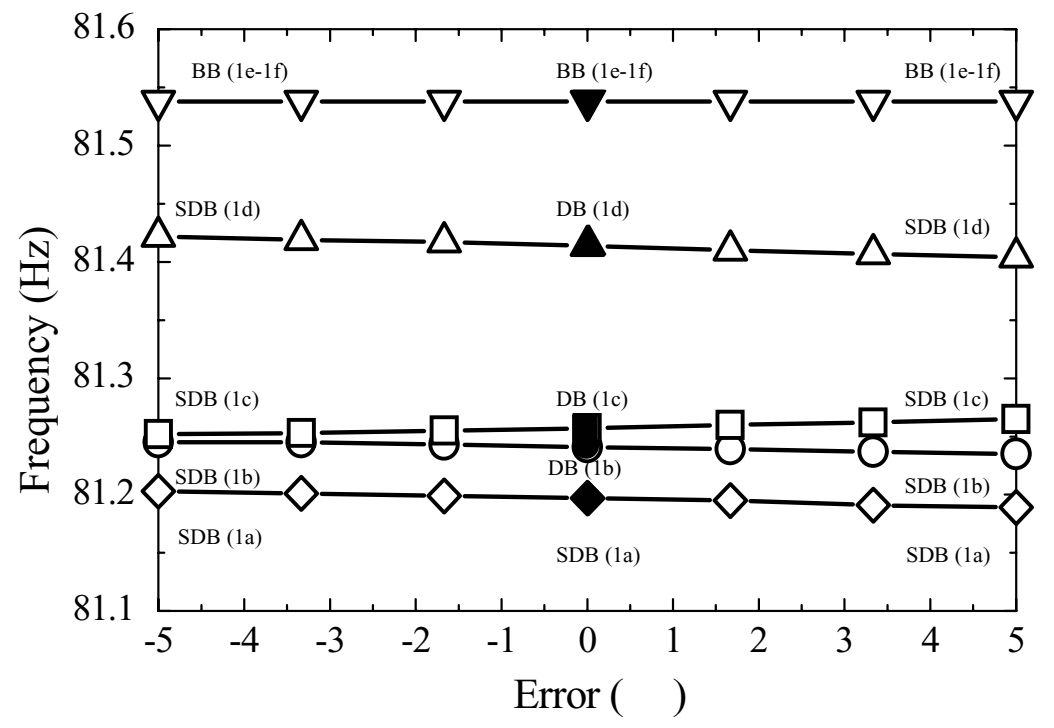

Fig. 8. Frequencies varying with the mistuned error for a six-blade rotor. 


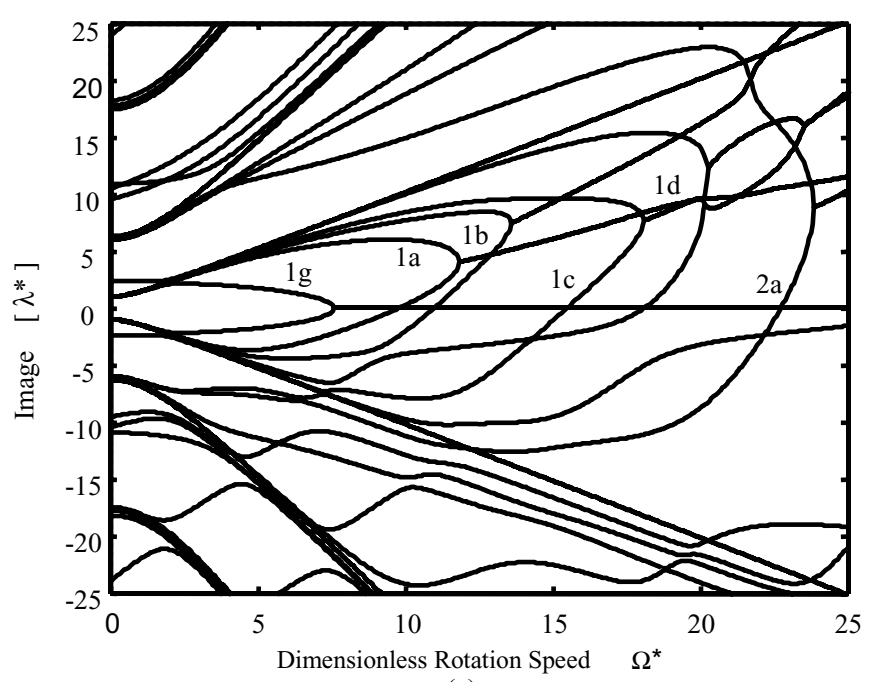

(a)

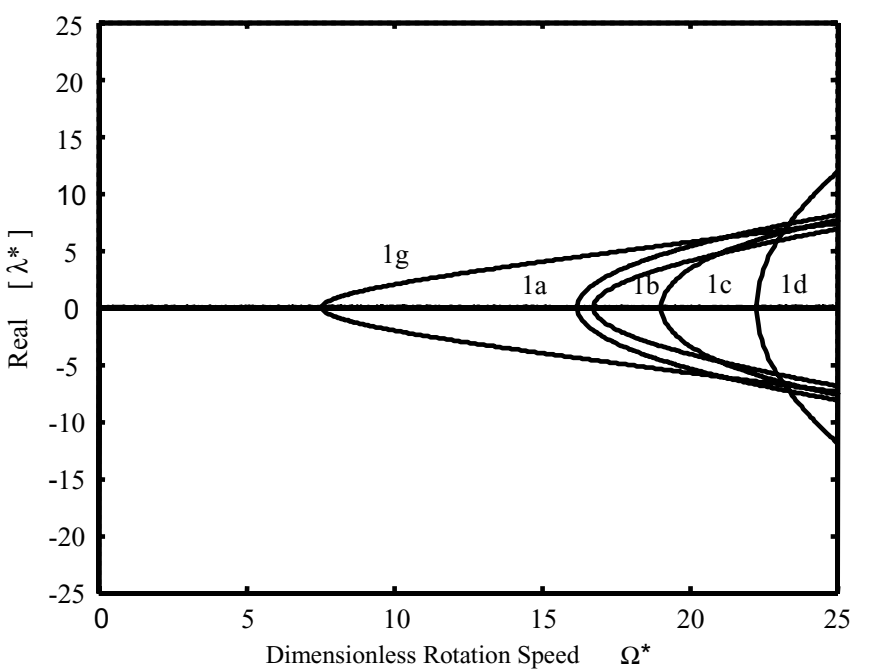

(b)

Fig. 9. Variation of eigenvalues with rotation speed for a well-tuned six-blade rotor

part of $\lambda$ varying with rotation. It is seen that $\operatorname{Re}(\lambda)$ is zero until the merging point, at which it becomes a pair of real numbers. It is seen that instability occurs first at $1 \mathrm{~g}$ mode, then $1 \mathrm{a}, 1 \mathrm{~b}, \ldots$ etc.

Figure 10 shows the same system but with one mistuned blade. Very interestingly, Fig. 10(b) shows instability exists only at the $1 \mathrm{~g}$ mode, in which the shaft's torsion dominates. In words, all the blade-dominating modes show no instability due to the mistuned blade destroying the periodic property. From the viewpoint, mistune by no means implies only disadvantages.

\section{Conclusions}

This paper explored the coupling modes of a shaft-disk-blade system with a mistuned blade's staggle angle. The study began with the modes evolvement resulting from a flexible shaft. It was discovered that in addition to the DB and BB modes, there existed SDB modes when the shaft was flexible.

A mistuned blade has drawn an important phenomenon on the coupling modes. The original DB modes existed no more, but replaced by SDB modes. It is due to the mistune destroying the balance between disk and blades. 


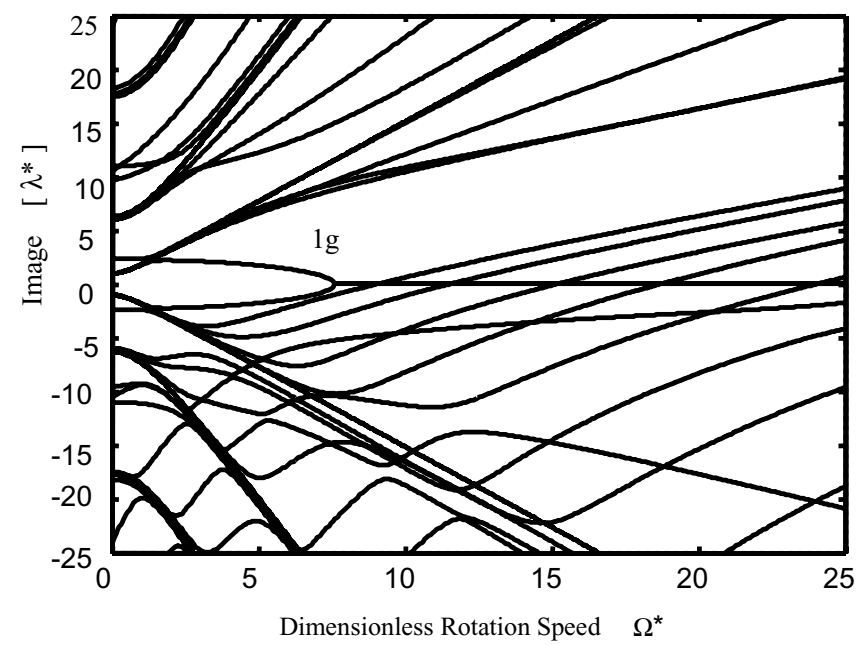

(a)

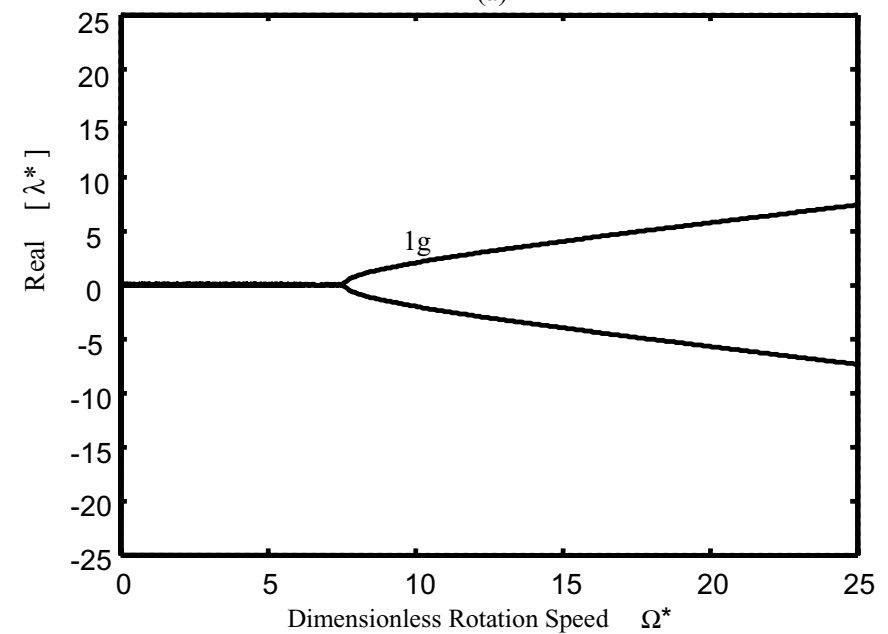

(b)

Fig. 10. Variation of eigenvalues with rotation speed for a mistuned six-blade rotor

The balance situation exists only at shaft-disk-blade (SDB) or blade-blade (BB) modes. Numerical calculation also revealed that the natural frequencies were affected by mistuned angle in an almost linear trend.

Finally, the effects of rotation on the system's natural frequencies were investigated. Frequency loci showed merge phenomenon with increasing rotational speed and eventually reached instability. A mistuned blade yet reduced some unstable possibilities caused by blade-dominating modes, which existed in a well-tuned rotor. The shaft-dominating mode was yet unaffected by the blade's mistune and retained possible instability. Form the results it is seen a mistuned blade might diminish the instability caused by blade-dominating modes.

\section{Appendix: Matrices elements}

$$
\beta_{k}= \begin{cases}\beta^{*}=\beta+\Delta \beta=\beta(1+\varepsilon), & k=1 \\ \beta, & k \neq 1\end{cases}
$$




$$
\begin{aligned}
& {\left[M_{s}\right]_{i j}=\int_{0}^{L s} I_{s} \Phi_{i} \Phi_{j} d Z+I_{d}\left[\Phi_{i} \Phi_{j}\right]_{Z=Z_{d}}+\rho_{b} A_{b} \sum_{1}^{N_{b}} \int_{r_{d}}^{r_{b}} x^{2}\left[\Phi_{i} \Phi_{j}\right]_{Z=Z_{d}} d x} \\
& {\left[M_{s b}\right]_{i j}=\left.\rho_{b} A_{b} \int_{r_{d}}^{r_{b}} x \Phi_{i}\right|_{Z=Z_{d}} V_{j} \cos \beta_{k} d x} \\
& {\left[M_{b}\right]_{i j}=\rho_{b} A_{b} \int_{r_{d}}^{r_{b}} V_{i} V_{j} d x} \\
& {\left[M_{d}\right]_{i j}=\rho_{d} h_{d}\left\{\int_{r_{s}}^{r_{d}} \int_{0}^{2 \pi} W_{i}^{s} W_{j}^{s} r d \theta d r+\int_{r_{s}}^{r_{d}} \int_{0}^{2 \pi} W_{i}^{c} W_{j}^{c} r d \theta d r\right\}}
\end{aligned}
$$

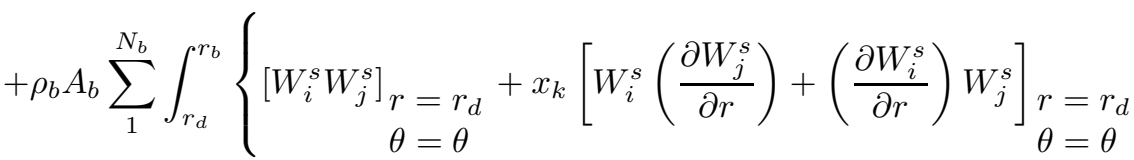

$$
\begin{aligned}
& \left.+x^{2}\left[\left(\frac{\partial W_{i}^{s}}{\partial r}\right)\left(\frac{\partial W_{j}^{s}}{\partial r}\right)\right] \begin{array}{c}
r=r_{d} \\
\theta=\theta
\end{array}\right\} d x \\
& +\rho_{b} A_{b} \sum_{1}^{N_{b}} \int_{r_{d}}^{r_{b}}\left\{\begin{array}{rl}
{\left[W_{i}^{c} W_{j}^{c}\right]_{r}=r_{d}} \\
\theta=\theta
\end{array}+x_{k}\left[W_{i}^{c}\left(\frac{\partial W_{j}^{c}}{\partial r}\right)+\left(\frac{\partial W_{i}^{c}}{\partial r}\right) W_{j}^{c}\right]_{\begin{array}{r}
r \\
\theta
\end{array}=r_{d}}\right. \\
& \left.+x^{2}\left[\left(\frac{\partial W_{i}^{c}}{\partial r}\right)\left(\frac{\partial W_{j}^{c}}{\partial r}\right)\right] \begin{array}{c}
r \\
\theta=r_{d} \\
\theta=\theta
\end{array}\right\} d x \\
& {\left[M_{d b}\right]_{i j}=-\rho_{b} A_{b}\left\{\int_{r_{d}}^{r_{b}}\left[W_{i}^{s}+x\left(\frac{\partial W_{i}^{s}}{\partial r}\right)\right]_{\substack{r=r_{d} \\
\theta=\theta}} V_{j} \sin \beta_{k} d x\right.} \\
& \left.+\int_{r_{d}}^{r_{b}}\left[W_{i}^{c}+x\left(\frac{\partial W_{i}^{c}}{\partial r}\right)\right]_{\substack{r \\
\theta=r_{d}}} V_{j} \sin \beta_{k} d x\right\} \\
& {\left[P_{d}^{s}\right]_{i j}=\rho_{d} h_{d}\left\{\int_{r_{s}}^{r_{d}} \int_{0}^{2 \pi}\left[W_{i}^{s}\left(\frac{\partial W_{j}^{s}}{\partial \theta}\right)+\left(\frac{\partial W_{i}^{s}}{\partial \theta}\right) W_{j}^{s}\right] r d \theta d r\right.} \\
& \left.+\int_{r_{s}}^{r_{d}} \int_{0}^{2 \pi}\left[W_{i}^{c}\left(\frac{\partial W_{j}^{c}}{\partial \theta}\right)+\left(\frac{\partial W_{i}^{c}}{\partial \theta}\right) W_{j}^{c}\right] r d \theta d r\right\} \\
& {\left[K_{s}^{e}\right]_{i j}=\int_{0}^{L s} G_{s} J_{s} \Phi_{i}^{\prime} \Phi_{j}^{\prime} d Z} \\
& {\left[K_{d}^{e}\right]_{i j}=D\left\{\int_{r_{s}}^{r_{d}} \int_{0}^{2 \pi} r\left(\nabla^{2} W_{i}^{s}\right)\left(\nabla^{2} W_{j}^{s}\right) d r d \theta+\int_{r_{s}}^{r_{d}} \int_{0}^{2 \pi} r\left(\nabla^{2} W_{i}^{c}\right)\left(\nabla^{2} W_{j}^{c}\right) d r d \theta\right\}} \\
& +2(1-\nu) D\left\{\int_{r_{s}}^{r_{d}} \int_{0}^{2 \pi} \frac{1}{r}\left[\left(\frac{\partial^{2} W_{i}^{s}}{\partial r \partial \theta}-\frac{1}{r} \frac{\partial W_{i}^{s}}{\partial \theta}\right)\left(\frac{\partial^{2} W_{j}^{s}}{\partial r \partial \theta}-\frac{1}{r} \frac{\partial W_{j}^{s}}{\partial \theta}\right)\right] d \theta d r\right.
\end{aligned}
$$




$$
\begin{aligned}
& \left.+\int_{r_{s}}^{r_{d}} \int_{0}^{2 \pi} \frac{1}{r}\left[\left(\frac{\partial^{2} W_{i}^{c}}{\partial r \partial \theta}-\frac{1}{r} \frac{\partial W_{i}^{c}}{\partial \theta}\right)\left(\frac{\partial^{2} W_{j}^{c}}{\partial r \partial \theta}-\frac{1}{r} \frac{\partial W_{j}^{c}}{\partial \theta}\right)\right] d \theta d r\right\} \\
& -(1-\nu) D\left\{\int_{r_{s}}^{r_{d}} \int_{0}^{2 \pi}\left[\left(\frac{\partial^{2} W_{i}^{s}}{\partial r^{2}}\right)\left(\frac{\partial W_{j}^{s}}{\partial r}+\frac{1}{r} \frac{\partial^{2} W_{j}^{s}}{\partial \theta^{2}}\right)+\left(\frac{\partial W_{i}^{s}}{\partial r}+\frac{1}{r} \frac{\partial^{2} W_{i}^{s}}{\partial \theta^{2}}\right)\left(\frac{\partial^{2} W_{j}^{s}}{\partial r^{2}}\right)\right] d \theta d r\right. \\
& \left.+\int_{r_{s}}^{r_{d}} \int_{0}^{2 \pi}\left[\left(\frac{\partial^{2} W_{i}^{c}}{\partial r^{2}}\right)\left(\frac{\partial W_{j}^{c}}{\partial r}+\frac{1}{r} \frac{\partial^{2} W_{j}^{c}}{\partial \theta^{2}}\right)+\left(\frac{\partial W_{i}^{c}}{\partial r}+\frac{1}{r} \frac{\partial^{2} W_{i}^{c}}{\partial \theta^{2}}\right)\left(\frac{\partial^{2} W_{j}^{c}}{\partial r^{2}}\right)\right] d \theta d r\right\} \\
& {\left[K_{b}^{e}\right]_{i j}=E_{b} I_{A} \int_{r_{d}}^{r_{b}} V_{i}^{\prime \prime} V_{j}^{\prime \prime} d x} \\
& {\left[K_{d}^{i}\right]_{i j}=h_{d}\left\{\int_{r_{s}}^{r_{d}} \int_{0}^{2 \pi}\left[\sigma_{r}\left(\frac{\partial W_{i}^{s}}{\partial r}\right)\left(\frac{\partial W_{j}^{s}}{\partial r}\right)+\frac{\sigma_{\theta}}{r^{2}}\left(\frac{\partial W_{i}^{s}}{\partial \theta}\right)\left(\frac{\partial W_{j}^{s}}{\partial \theta}\right)\right] d \theta d r\right.} \\
& \left.+\int_{r_{s}}^{r_{d}} \int_{0}^{2 \pi}\left[\sigma_{r}\left(\frac{\partial W_{i}^{c}}{\partial r}\right)\left(\frac{\partial W_{j}^{c}}{\partial r}\right)+\frac{\sigma_{\theta}}{r^{2}}\left(\frac{\partial W_{i}^{c}}{\partial \theta}\right)\left(\frac{\partial W_{j}^{c}}{\partial \theta}\right)\right] d \theta d r\right\} \\
& {\left[K_{s}^{\Omega}\right]_{i j}=\frac{1}{2} \rho_{b} A_{b} \sum_{1}^{N_{b}} \int_{r_{d}}^{r_{b}}\left(r_{b}^{2}-3 x^{2}\right)\left[\Phi_{i} \Phi_{j}\right]_{Z=Z_{d}} d x} \\
& {\left[K_{s b}^{\Omega}\right]_{i j}=\left.\rho_{b} A_{b} \int_{r_{d}}^{r_{b}} x \Phi_{i}\right|_{Z=Z_{d}} V_{j} \cos \beta_{k} d x-\left.\frac{1}{2} \rho_{b} A_{b} \int_{r_{d}}^{r_{b}}\left(r_{b}^{2}-x^{2}\right) \Phi_{i}\right|_{Z=Z_{d}} V_{j}^{\prime} \cos \beta_{k} d x} \\
& {\left[K_{d}^{\Omega}\right]_{i j}=\rho_{d} h_{d}\left\{\int_{r_{s}}^{r_{d}} \int_{0}^{2 \pi}\left(\frac{\partial W_{i}^{s}}{\partial \theta}\right)\left(\frac{\partial W_{j}^{s}}{\partial \theta}\right) r d \theta d r+\int_{r_{s}}^{r_{d}} \int_{0}^{2 \pi}\left(\frac{\partial W_{i}^{c}}{\partial \theta}\right)\left(\frac{\partial W_{j}^{c}}{\partial \theta}\right) r d \theta d r\right\}} \\
& {\left[K_{b}^{\Omega}\right]_{i j}=\rho_{b} A_{b} \int_{r_{d}}^{r_{b}} V_{i} V_{j} \cos ^{2} \beta_{k} d x-\frac{1}{2} \rho_{b} A_{b} \int_{r_{d}}^{r_{b}}\left(r_{b}^{2}-x^{2}\right) V_{i}^{\prime} V_{j}^{\prime} d x}
\end{aligned}
$$

\section{References}

[1] J.A. Dopkin and T.E. Shoup, Rotor Resonant Speed Reduction Caused by Flexibility of Disks, ASME J Engineering for Industry 96 (1974), 1328-1333.

[2] C.D. Mote, Free Vibrations of Initially Stressed Circular Disks, ASME J Engineering for Industry 87 (1965), $258-264$.

[3] I.Y. Shen, Closed-Form Forced Response of a Damped, Rotating, Multiple Disks/ Spindle System, ASME J Applied Mechanics 64 (1997), $343-352$.

[4] K.A. Ansari, On the Importance of Shear Deflection, Rotatory Inertia, and Coriolis Forces in Turbines Blade Vibrations, ASME $J$ Engineering for Gas Turbines and Power 108 (1986), 319-324.

[5] A.W. Leissa, J.C Macbain and R.E. Kielb, Vibrations of Twisted Cantilevered Plates-Summary of Previous and Current Studies, J Sound and Vibration 96(2) (1984), 159-173.

[6] M. Swaminathan and J.S. Rao, Vibration of Rotating, Pretwisted and Tapered Blades, Mechanism and Machine Theory 12 (1977), 331-337.

[7] R.L. Eshleman and R.A. Eubanks, On the Critical Speeds of a Continuous Shaft-Disk System, ASME J Engineering for Industry 89 (1967), 645-652.

[8] R.M. Laurenson, Modal Analysis of Rotating Flexible Structure, AIAA Journal 14(10) (1976), 1444-1450.

[9] H.N. Ozguven, On the Critical Speed of Continuous Shaft-Disk Systems, ASME J Vibration and Acoustics 106 (1984), 59-61.

[10] D.J. Ewins, Vibration Characteristics of Bladed Disc Assemblies, J Mechanical Engineer Science 15(3) (1973), 165-185.

[11] F. Kushner, Disc Vibration-Rotating Blade and Stationary Vane Interaction, J Mechanical Design 102 (1980), 579-584.

[12] V. Omprakash and V. Ramamurti, Analysis of Bladed Disks - a Review, Shock and Vibration Digest 11 (1988), 14-21.

[13] D.R. Chivens and H.D. Nelson, The Natural Frequencies and Critical Speeds of a Rotating, Flexible Shaft-Disk System, ASME $J$ Engineering for Industry 97 (1975), 881-886.

[14] J.S. Chen and C.C. Wong, Vibration and Stability of a Spinning Disk in Contact With Evenly-Spaced Stationary Load Systems, ASME J Vibration and Acoustics 120 (1998), 301-303.

[15] F. Wu and G.T. Flowers, A Transfer Matrix Technique for Evaluating the Natural Frequencies and Critical Speeds of a Rotor with Multiple Flexible disks, ASME J Vibration and Acoustics 114 (1992), 242-248. 
[16] C.W. Lee and S.B. Chun, Vibration Analysis of a Rotor With Multiple Flexible Disks Using Assumed Modes Method, ASME J Vibration and Acoustics 120 (1998), 87-94.

[17] V. Karadog, Finite Element Dynamic Analysis of Blade Shear Centre Effects on Practical Blades Disks, J Sound and Vibration 94 (1984), $183-197$.

[18] C.A.M. Soares and M. Petyt, Finite Element Dynamic Analysis of Practical Bladed Disks, J Sound and Vibration 61 (1978), 561-570.

[19] V. Omprakash and V. Ramamurti, Natural Frequencies of Bladed Disks by a Combined Cyclic Symmetry and Rayleigh-Ritz Method, $J$ Sound and Vibration 125(2) (1988), 357-366.

[20] S.B. Chun and C.W. Lee, Vibration of Shaft-Bladed Disk System by Using Substructure Synthesis and Assumed Modes Method, J Sound and Vibration 189 (1996), 587-608.

[21] S.C. Huang and K.B. Ho, Coupled Shaft-Torsion and Blade-Bending Vibration of a Rotation Shaft-Disk-Blade Unit, ASME J Engineering for Gas Turbines and Power 118 (1996), 100-106.

[22] C.H. Yang and S.C. Huang, Coupling Vibration in Rotating Shaft-Disk-Blades System, ASME J Vibration and Acoustics. Accepted.

[23] S.C. Huang and W.J. Chiou, Modeling and Vibration Analysis of Spinning-Disk and Moving-Head Assembly in Computer Storage Systems, ASME Journal of Vibration and Acoustics 119 (1997), 185-191. 

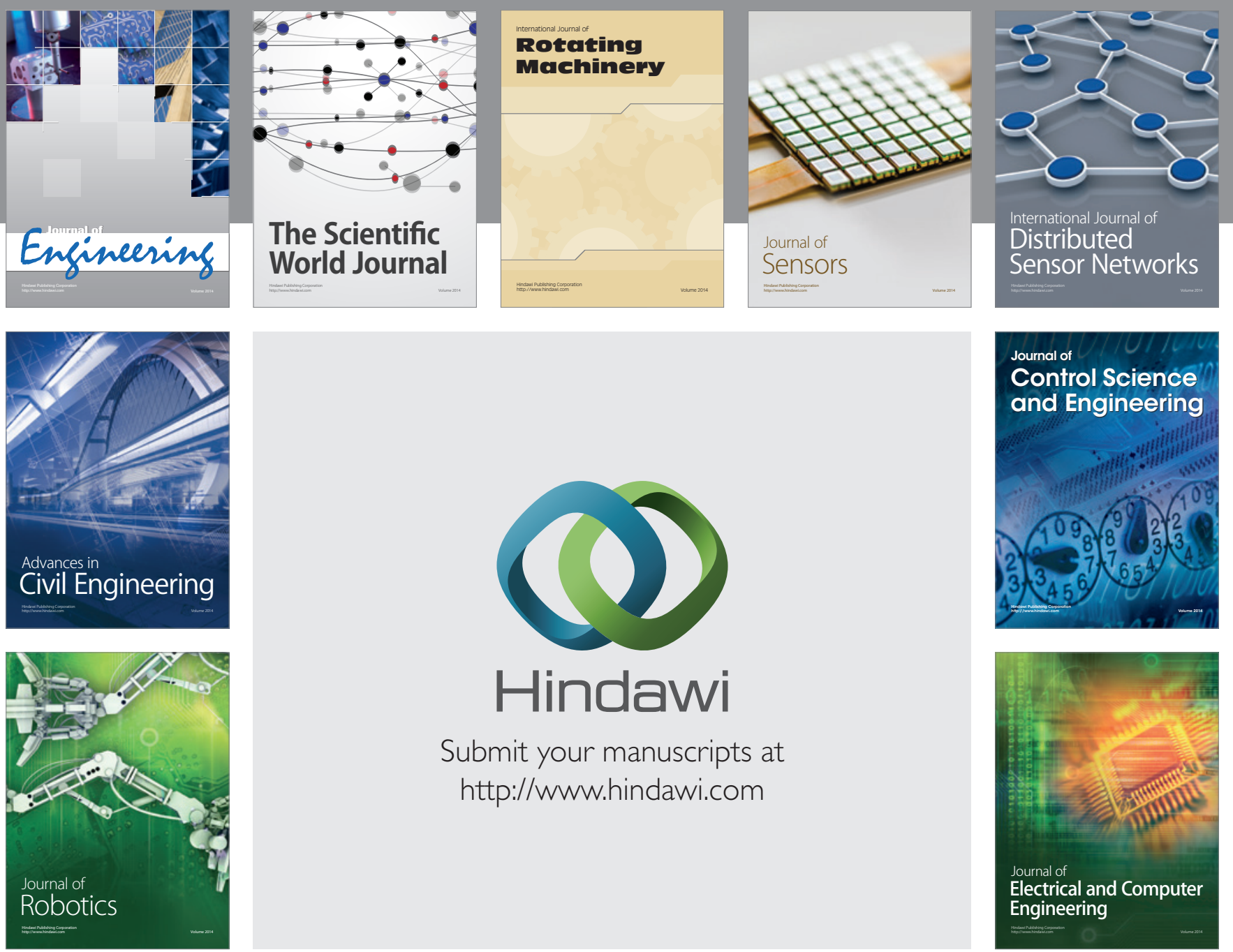

Submit your manuscripts at

http://www.hindawi.com
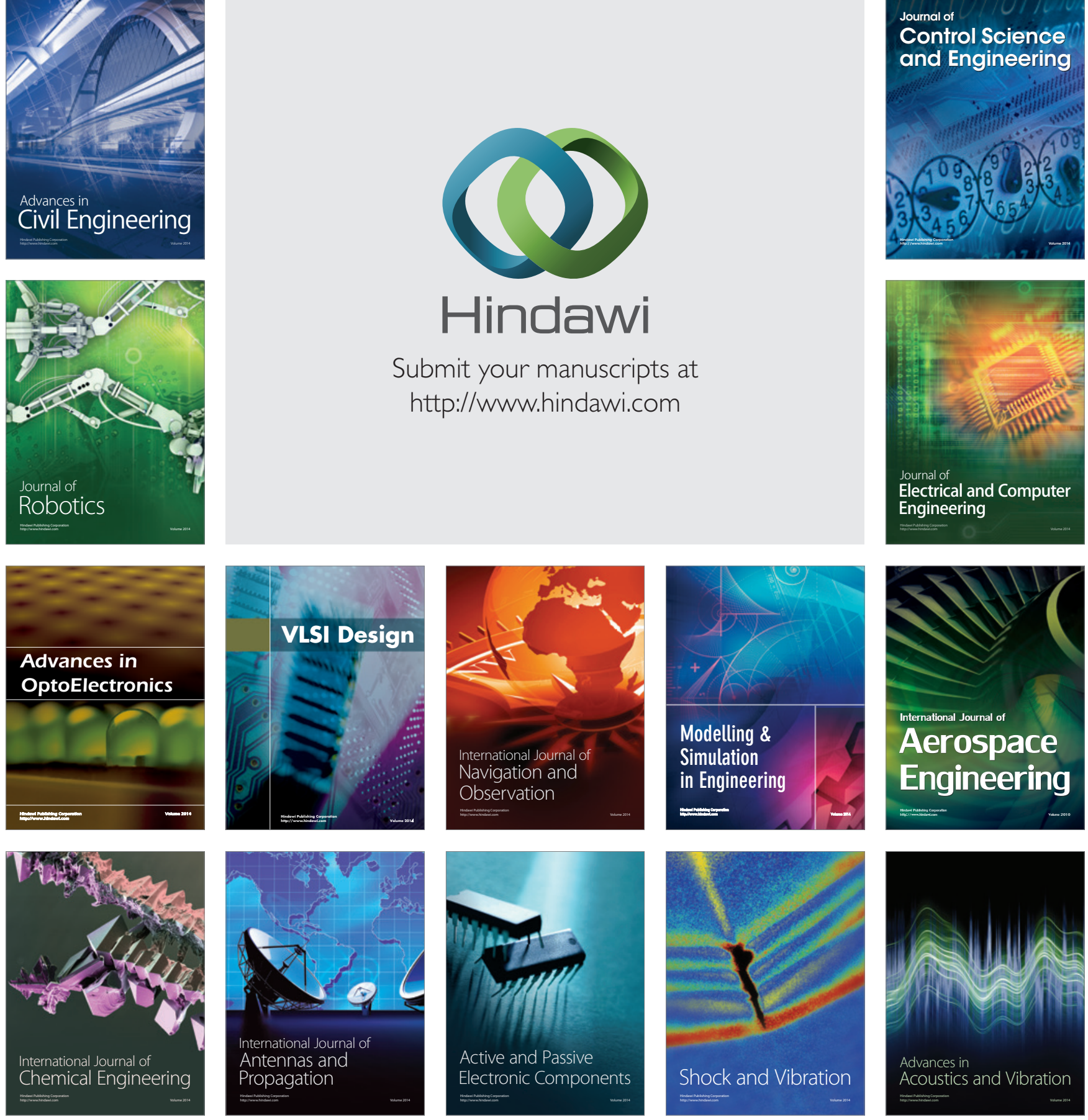